\title{
How number-space relationships are assessed before formal schooling: A taxonomy proposal
}

\author{
Katarzyna Patro ${ }^{1,2}$, Hans-Christoph Nuerk ${ }^{2,3}$, Ulrike Cress ${ }^{2}$ and Maciej Haman ${ }^{1}$ \\ 1 Department of Psychology, University of Warsaw, Warsaw, Poland \\ ${ }^{2}$ Knowledge Construction Lab, Knowledge Media Research Center, Tübingen, Germany \\ ${ }^{3}$ Department of Psychology, University of Tübingen, Tübingen, Germany
}

\section{Edited by:}

Natasha Kirkham, Birkbeck College,

UK

Reviewed by:

Natasha Kirkham, Birkbeck College,

UK

Daniel Richardson, University

College London, UK

\section{*Correspondence:}

Katarzyna Patro, Knowledge

Construction Lab, Knowledge Media

Research Center, Schleichstrasse 6,

72076 Tübingen, Germany

e-mail: katarzyna.patro@gmail.com
The last years of research on numerical development have provided evidence that spatial-numerical associations (SNA) can be formed independent of formal school training. However, most of these studies used various experimental paradigms that referred to slightly different aspects of number and space processing. This poses a question of whether all SNAs described in the developmental literature can be interpreted as a unitary construct, or whether they are rather examples of different, but related phenomena. Our review aims to provide a starting point for a systematic classification of SNA measures used from infancy to late preschool years, and their underlying representations. We propose to distinguish among four basic SNA categories: (i) cross-dimensional magnitude processing, (ii) associations between spatial and numerical intervals, (iii) associations between cardinalities and spatial directions, (iv) associations between ordinalities and spatial directions. Such systematization allows for identifying similarities and differences between processes and representations that underlie the described measures, and also for assessing the adequacy of using different SNA tasks at different developmental stages.

Keywords: infants, number, preschoolers, space, SNA, SNARC, taxonomy

\section{INTRODUCTION}

Over the last 20 years, different experimental paradigms have been employed to examine mental connections between numbers and space. For instance, a parity judgment task with bimanual responses usually reveals the SNARC effect (Spatial-Numerical Associations of Response Codes) which is defined as left-sided response advantage for smaller numbers, and right-sided for larger numbers (Dehaene et al., 1993). This and other kinds of spatial-numerical associations (SNA) were originally thought to be acquired through a long-term experience in a certain cultural environment with specific script direction and formal school training. However, this late development account has been recently challenged because some forms of numberspace associations have been discovered to develop before formal schooling.

A closer look reveals that the various SNA investigations in young children have referred to different associations between numbers and space. In fact, the term "space" in number-space relationship is understood and assessed in a variety of ways. Sometimes its directionality is examined (left-right dimension), whereas in other studies its non-directional extension (number lengths). The term "number" has also been used with different connotations: Some studies referred to ordinality (counting) and others to cardinality (numerical magnitudes).

In sum, several theoretical and methodological differences between SNA tasks exist in studies of children, which may address different underlying space and number representations. To date, all SNA studies have been lumped into one category, but no systematic distinction of early SNA measures exists. In the current review, a systematic taxonomy is proposed to enable classifying present and future approaches to research on SNA before school education.

\section{DISTINGUISHING DIFFERENT SNA EFFECTS IN PRESCHOOLERS AND INFANTS: TOWARD A TAXONOMY}

While many comprehensive reviews have described varieties of SNAs and their assessments in adults (Gevers and Lammertyn, 2005; Hubbard et al., 2005; Wood et al., 2008), there is only one theoretical article on the pre-linguistic basis of the number-space link (de Hevia et al., 2012), which includes a short overview about developmental studies. While this review is ground-breaking in that it establishes early SNA as a widespread phenomenon, it does not provide a systematization of employed tasks, nor the underlying representations assessed in different age ranges. In the following paragraphs, we emphasize the reasons why a systematization of SNA tasks is important, especially for developmental studies.

First, it is a classical question in psychology, whether different tasks used to assess an underlying construct measure the same construct. The broader the construct is, the more critical this question becomes. The SNA is an example of such a broad construct. Different number representations and their components can be related to space (non-symbolic numerosities, symbolic magnitude, position in a sequence), and different aspects of space can be studied in relation to number processing (length, directionality). In adult studies, not all of these components comprising number-space associations activate the same neurocognitive representations (Turconi et al., 2004; Cohen 
Kadosh et al., 2007). Therefore, these components should be systematically distinguished.

Second, adult reviews are not sufficient, because different SNAs might emerge at different developmental stages, as different aspects of numerical or spatial information are accessible at different ages. Therefore, general conclusions from adult SNA paradigms may fall short, because the numerical or spatial information employed is inaccessible to young children.

For these reasons, in this review we outline systematic distinctions between SNA effects and their assessment in children before school age. We show that the methods used so far might be based on different aspects of number and space processing-on directional and non-directional space representation, and the representation of cardinal, ordinal or interval numerical information. Because this variety of representations might lead to different ways of defining a number-space link, we suggest distinguishing among at least four main SNA categories, as a first step. Two of them refer to a non-directional number-space mapping: (i) crossdimensional magnitude processing, and (ii) associations between spatial and numerical intervals. The other two categories refer to directional number-space mapping: (iii) associations between cardinalities and spatial directions, and (iv) associations between ordinalities and spatial directions.

The order of these categories does not reflect the order in which they arise across lifespan. As will be shown later, investigations within some of these categories are focused mostly on one age group and are based on similar experimental paradigms, developed by one or two research groups. Therefore, the current state of research does not allow making strong conclusions about the origins and developmental trajectory of SNA.

Furthermore, we are aware that other distinctions beyond these four categories are possible. For instance, one might distinguish between symbolic and non-symbolic numerosities. However, although symbolic numbers have been employed in some studies reported here, using them as stimuli might be sometimes misleading because only the oldest children master them reliably (see the discussion of the last category). Therefore, we have constrained our categories to those distinctions that have played a major role in prior research on SNA in young children. Thus, our four categories provide a starting point based on the research, but are not meant to preclude additional distinctions that might become relevant in future studies.

Finally, for most of these tasks, it is discussed whether the observed number-space associations are caused by respective representations or rather by corresponding task properties. Although it is beyond a scope of this review to discuss thoroughly all the critics, we will refer to them shortly to provide the reader with a critical overview of the SNA research.

In the next sections, we provide a short outline of assessments and representations tested in each SNA category, the age of the participants with which they have been applied, and describe specific features of the representations and their underlying processes. We also briefly refer to certain controversies aroused by some of these measures. This systematization is summarized in Table 1.

\section{SNA CATEGORY 1: CROSS-DIMENSIONAL MAGNITUDE PROCESSING \\ MEASURES}

Extraction of an abstract rule across spatial-numerical dimensions (de Hevia and Spelke, 2010; Lourenco and Longo, 2010). Eightand nine-month-old infants were habituated to an abstract rule referring either to numerosity or to length (e.g., color pattern cues assigned to a certain magnitude or ascending/descending orders of magnitude sequences). In a testing phase, a magnitude dimension presented during habituation (e.g., numerosity) was replaced by another dimension (e.g., length). Despite this change, infants detected if an abstract rule, to which they were habituated, had been reversed.

Numerosity-length matching (de Hevia and Spelke, 2010; de Hevia et al., 2012). Four-year-old preschoolers were presented with a rule of a positive magnitude matching (larger numerosities paired with longer lines), or an inverse rule (larger-shorter pairings). Most of the children found a correct match in the testing trials, but only in the positive mapping condition. Similarly, following a short familiarization to larger-numerosity-longer-line pairings, 8-month-old infants demonstrated a looking time preference for novel stimuli matched according to the same, recently acquired rule. A familiarization to an inverse rule did not cause any preference in later trials.

Line bisection (de Hevia and Spelke, 2009). Five-year-old children were instructed to mark the center of a horizontal line flanked by two arrays of dots with different numerosities. Children placed the midpoint of a line closer to a larger numerosity side.

\section{MAIN FEATURES OF THE SNA CATEGORY 1}

The above tasks used different experimental paradigms to explore interrelations between cardinal aspects of non-symbolic numerosities and non-directional spatial dimension - line length. They indicate that length and numerosity are related even for infants and young children, either in a complementary way, carrying together a common abstract rule, or in an interfering way, when a value of one dimension modulates processing of another dimension.

Such kinds of interactions can be explained by theories postulating a generalized system for processing magnitudes of different sorts (Walsh, 2003; Cantlon et al., 2009). Processing of length, number, and other magnitude dimensions share many representational features, for example a continuous and quantitative metric or early ontogenetic and evolutionary beginnings. Therefore, it is postulated that the human mind is predisposed to form a common representational framework for numerical and spatial quantities, or at least to map one dimension onto another. As we will see, this makes the numerosity-length bond unique and qualitatively different from the other SNA categories, for which an influence of cultural factors has been postulated or demonstrated.

These methods are also critically discussed. When stimuli consist of object's collections, certain perceptual set features (density, elements' size) correlated with numerosities may drive participants' choices. For instance, Gebuis and Gevers (2011) challenged the numerical explanation of the line bisection bias. In their experiment, when numerosity was negatively correlated with a 
Table 1 | The four distinguished SNA categories, their measures, studies in which these measures were used, and mean age of participants.

\begin{tabular}{|c|c|c|c|}
\hline $\begin{array}{l}\text { Category of spatial-numerical } \\
\text { associations (SNA) }\end{array}$ & Task $^{\mathbf{a}}$ & Study & $\begin{array}{l}\text { Mean age (average ages provided as decimal } \\
\text { fractions have been recalculated to a } \\
\text { "years-months-days" format) }\end{array}$ \\
\hline
\end{tabular}

SNA1: Cross-dimensional magnitude processing

Space: non-directional Number: cardinal
Extraction of an abstract rule across spatial-numerical dimensions
Lourenco and Longo, 2010

de Hevia and Spelke, 2010, Exp. 1
$M=0$ year 9 months 4 days

$M=0$ year 8 months
Numerosity-length matching

de Hevia and Spelke, 2010, Exp. 2, 3 de Hevia et al., 2012; $\begin{aligned} M & =0 \text { year } 8 \text { months } 3 \text { days (Exp. 2), } \\ M & =0 \text { year } 8 \text { months } 2 \text { days (Exp. } 3 \text { ) }\end{aligned}$

$M=4$ years 1 month
Line bisection

Number line task de Hevia and Spelke, 2009, Exp. 4, 5,6
$M=5$ years 1 months (Exp. 4), $M=4$ years 11 months (Exp. 5), $M=4$ years 9 months (Exp. 6)
SNA2: Associations between spatial and numerical intervals
Siegler and Booth, 2004, Exp. 1, 2

Booth and Siegler, 2006, Exp. 1

Ebersbach et al., 2008

Berteletti et al., 2010, Exp. 1, 2
$M=5$ years 10 months (Exp. 1), $M=6$ years 1 month (Exp. 2)

$M=5$ years 8 months

$M=5$ years 3 months

$M_{1}=4$ years 0 month, $M_{2}=5$ years 0 month,

$M_{3}=5$ years 11 months (Exp. 1)

$M_{1}=4$ years 0 month, $M_{2}=4$ years

11 months, $M_{3}=5$ years 10 months (Exp. 2)

$M=5$ years 7 months

$M=5$ years 10 months

$M_{1}=4$ years 6 months, $M_{2}=5$ years 2 months

$M=5$ years 11 months

$M=6$ years 0 month
Space: non-directional ${ }^{\mathrm{b}}$

Number: interval
Fischer et al., 2011

Muldoon et al., 2011

Slusser et al., 2013, Exp. 1

Ebersbach, in press

Patro and Haman, 2012

Hoffmann et al., 2013

SNARC tasks

Ebersbach et al., 2013

Opfer and Thompson, 2006 (Study1)
SNA4: Associations between

ordinalities and spatial directions

Counting

Opfer and Furlong, 2011

Shaki et al., 2012, Exp. 2

Addition/removal of one object

Space: directional

Number: ordinal

Addition/removal of one object

Opfer and Furlong, 2011

Spatial search
Opfer et al., 2010, Exp. 1

Opfer and Furlong, 2011
$M=4$ years 0 month

$M_{1}=5$ years 6 months, $M_{2}=5$ years

10 months

$M=5$ years 11 months

$M_{1}=3$ years 3 months, $M_{2}=4$ years 0 month, $M_{3}=5$ years 2 months

$M_{1}=4$ years 6 months, $M_{2}=4$ years

6 months, $M_{3}=4$ years 6 months, $M_{4}=4$ years 5 months

$M_{1}=4$ years 2 months, $M_{2}=4$ years

1 months, $M_{3}=3$ years 11 months

Opfer and Thompson, 2006 (Study1) $M_{1}=3$ years 3 months, $M_{2}=4$ years 0 ,

$M_{3}=5$ years 2 months

$M_{1}=4$ years 6 months, $M_{2}=4$ years

5 months, $M 3=4$ years 6 months, $M_{4}=4$ years 5 months

$M_{1}=4$ years 6 months, $M_{2}=4$ years 6 months

$M_{1}=4$ years 6 months, $M 2=4$ years

6 months, $M_{3}=4$ years 6 months, $M_{4}=4$ years 5 months

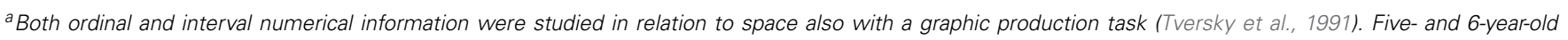

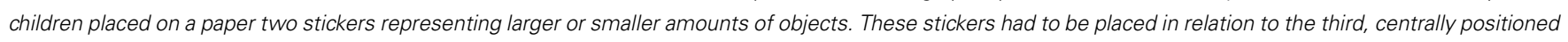
sticker that represented a medium amount of the same things. However, interval relations between numerosities were not mapped by children as spatial distances, and there were no consistent directional biases.

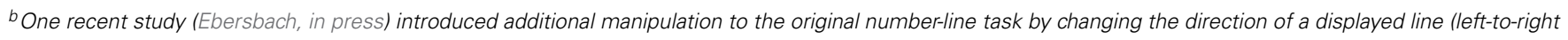

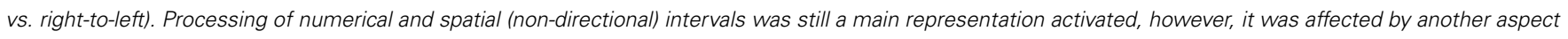

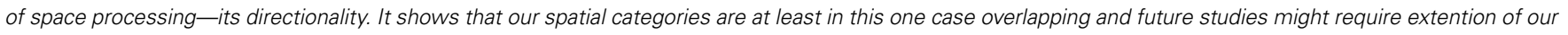
taxonomy.
} 
set's area, a bias occurred toward a smaller numerosity (larger area). They argued that the bisection bias was visually rather than numerically driven. However, several arguments against their critics were also presented (de Hevia, 2011). Whether children process numerosities independently of perceptual cues becomes now an intensively discussed issue (Cantrell and Smith, 2013; Szúcs et al., 2013; Libertus et al., 2014 for opposite views), which will certainly trigger more studies addressing this question.

\section{SNA CATEGORY 2: ASSOCIATIONS BETWEEN SPATIAL AND NUMERICAL INTERVALS \\ MEASURE}

Number-line task (Siegler and Booth, 2004; Booth and Siegler, 2006; Ebersbach et al., 2008; Berteletti et al., 2010; Barth and Paladino, 2011; Fischer et al., 2011; Muldoon et al., 2011; Ebersbach, in press). Preschoolers (from the age of 4 and older) were instructed to place a target number on a line with 0 or 1 at one end, and 10,20, 100 or other numbers at the second end (depicted in Arabic or non-symbolic format). Lower accuracy in younger children has been observed, characterized by overestimation of interval distances between smaller numbers (logarithmic scaling). Accuracy in this task increases with age and is correlated with other numerical capabilities.

\section{MAIN FEATURES OF THE SNA CATEGORY 2}

This task requires representation of interval scaling instead of cardinal magnitudes. If it is solved correctly, the interval of the numerical magnitude is isomorphic to spatial magnitude of the presented line. Thus, space representation activated here is nondirectional (like in SNA1).

A second important feature is that this task enforces spatial mapping of numbers. Thus, its primary purpose should be distinguished from the purposes of SNA1 measures: It does not evaluate whether numbers can be spontaneously related to space, but rather whether such a relationship, if it is induced, is constructed in a systematic interval-scaled way.

The idea that the estimations of the number-line task reflect scaling of a mental number line representation in an isomorphic way (Siegler and Opfer, 2003) has been criticized by various authors. Some authors argue that a logarithmic fit is produced by multi-linear representations for different number ranges (Ebersbach et al., 2008; Moeller et al., 2009). Other suggest that different proportional strategies are applied to solve the task (Barth and Paladino, 2011). The mental processes and strategies underlying the number-space associations in this task are thus still controversial.

\section{SNA CATEGORY 3: ASSOCIATIONS BETWEEN CARDINALITIES AND SPATIAL DIRECTIONS \\ MEASURE}

Numerosity comparison (Patro and Haman, 2012). Four-years-old children compared numerosities of two bilaterally presented sets. Reactions were faster when a smaller set was on the left side, and a larger one on the right side.

SNARC tasks (Ebersbach et al., 2013; Hoffmann et al., 2013). Five-years-old children classified a single number (or set of dots) between two categories (red/green, smaller/larger than $\mathrm{x}$ ) using left and right buttons. Left-sided reactions were faster to smaller numbers, and right-sided to larger numbers.

\section{MAIN FEATURES OF THE SNA CATEGORY 3}

SNA3 and SNA4 differ in a major way from SNA1 and 2, because these associations are about spatial directionality and not about non-directional spatial magnitude. Larger numbers are generally associated with one side of horizontal space (the right side in Western culture), while smaller numbers are associated with the other. In the above tasks, such a directional representation is formed for cardinal numbers.

There are at least two critical issues for this category. First, it is controversial whether an adult-like association is built between numerical and spatial representations (Dehaene et al., 1993), or rather between "smaller/larger" and "left/right" verbal categories (Nuerk et al., 2004; Proctor and Cho, 2006; Gevers et al., 2010). Which kind of association preschoolers build, has not yet been examined. Second, reading direction was traditionally believed to be a main source of SNARC (Zebian, 2005; Shaki and Fischer, 2008), whereas other not determined pre-literate factors may build SNA in preschoolers. If such factors are among early acquired cultural skills, it would strengthen the difference between SNA1 and SNA3 because the first category may be more innately determined.

\section{SNA CATEGORY 4: ASSOCIATIONS BETWEEN ORDINALITIES AND SPATIAL DIRECTIONS \\ MEASURES}

Counting (Opfer and Thompson, 2006; Opfer and Furlong, 2011; Shaki et al., 2012). English-, Hebrew-, and Arabic-speaking preschoolers (age 3-6) were asked to count objects aligned horizontally in a row. Most of English-speaking children counted from left to right, whereas Hebrew- and Arabic-speaking children counted from right to left.

Addition/removal of one object (Opfer and Thompson, 2006; Opfer and Furlong, 2011). In an addition task, English-speaking preschoolers (age 3-5) were presented with three objects aligned in a row, and asked to add one object. In a subtraction task, they were presented with four objects and asked to remove one of them. The number of participants who added to and removed from the correct right end increased with age, but it was still below 50\%.

Spatial search (Opfer et al., 2010; Opfer and Furlong, 2011). Four-year-old English-speaking children were presented with two boxes containing seven compartments, numbered in a left-toright or right-to-left order. An experimenter showed a hidden star in one compartment of the first box, and asked children to find a similar star in the second box, in the compartment with the same number. Children performed more accurately in a condition when compartments were numbered from left to right than in a condition with the reversed numerical order.

\section{MAIN FEATURES OF THE SNA CATEGORY 4}

The tasks from this category are based on directional processing of numerical orders. Ordering tasks may be subdivided into two categories: One category without necessary access to magnitude (e.g., counting tasks) and another category, in which 
order necessarily involves magnitude access or cardinality. For instance, ascending/descending numerosity-length matching (de Hevia and Spelke, 2010) requires some sort of order representation which is based on magnitude (smaller comes before larger). Therefore, in this category, we suggest to include only those studies in which a position of a number in a sequence (four comes before five, rightmost element should be removed), and not its magnitude, is relevant for solving a task.

Research with adults suggests that ordinal and cardinal processing of numbers rely at least partly on different processes. For instance, neural activation observed during classification of numbers as smaller or larger than a target has a different spatiotemporal pattern than during classifying them as coming before or after a target (Turconi et al., 2004). There is a strong agreement that both kinds of numerical processes constitute a number semantics (Sury and Rubinsten, 2012), but their contributions to building a numerical representation, especially in children, might be different.

In young preschoolers, the fixed order of a counting list is not primarily a numerical representation. Access to numerical semantics through symbolic notation establishes itself through the whole preschool period (Wynn, 1990; Le Corre and Carey, 2007). Although 3-year-olds might recite sequences of number words, they are usually not yet able to assign all learned numerals to corresponding values or to use a counting procedure to establish a set numerosity.

These considerations make the conceptual distinction between cardinal tasks (SNA3) and ordinal tasks (SNA4) valid, but a distinction does not imply that ordinal and cardinal knowledge are unrelated - the fixed order of a counting list may provide a scaffold for later understanding of cardinality. According to the cardinal principle of counting, the magnitude of the last ordinal element equals the cardinality. Children realize that quite late, usually only around the age of four. Once children have realized the relation between symbolic number order and number cardinality, however, order tasks like a counting or digit search task may become theoretically ambiguous, because it is sometimes not entirely clear to what extent ordinal and cardinal aspects of numbers contribute to the observed spatial associations.

Another important feature of SNA4 refers to its validity. In the tasks from this category, object stimuli are already arranged spatially forming a row. Thus, alike in SNA2, and contrary to SNA1 and 3, this task induces spatial coding and cannot evidence spontaneous number-space mapping. Note, however, that only a spatial location of response is enforced, but not directionality, because children can also count or subtract from the middle.

\section{SUMMARY}

In the taxonomy proposed here, we have distinguished four basic categories of SNA research in children before school age. Some of them share basic features, like directional components, or using a spatial magnitude as a basic mapping metric, but they differ at the same time in other aspects (processing ordinal, cardinal or interval number magnitudes).

These four distinguished SNA categories, although based on different number and space representations, are not intended to be exclusive. Our aim was to accentuate that although they are overlapping and related to each other, their basic mechanisms, developmental trajectory, and susceptibility to cultural experience might be different.

These categories are a starting point for categorizing the most important distinctions in current preschool SNA research. They may not be comprehensive for all future results. For instance, we have outlined that the distinction between symbolic and non-symbolic magnitudes is not of major importance in prior developmental studies on SNA, but it is possible that future studies will bring new insights about the symbol-grounding problem that will require extension of this taxonomy.

Developmental studies on SNA are still sparse and other research paradigms might bring new insights into individual trajectories, new distinctions, and the causal relations of different number-space associations. They could also help to clarify the issues of validity of different SNA measures. However, we believe that the current taxonomy helps to understand that the growing evidence about SNA before schooling is not unitary, and should be categorized with consideration of the major distinctions of underlying space and number concepts used in empirical studies to date.

\section{ACKNOWLEDGMENTS}

This work was supported by the National Science Center (NCN, Poland) grant no. DEC-2011/03/N/HS6/03095 to Katarzyna Patro, no. DEC-2012/05/B/HS6/03713 to Maciej Haman, by the German Research Foundation (DFG, Germany), grant no. CR110/8-1 to Ulrike Cress and Hans-Christoph Nuerk, and the excellence graduate school LEAD: Learning Educational Achievement and Life-Course Development supporting the work of Hans-Christoph Nuerk and Ulrike Cress.

\section{REFERENCES}

Barth, H. C., and Paladino, A. M. (2011). The development of numerical estimation: Evidence against a representational shift. Dev. Sci. 14, 125-135. doi: 10.1111/j.1467-7687.2010.00962.x

Berteletti, I., Lucangeli, D., Piazza, M., Dehaene, S., and Zorzi, M. (2010). Numerical estimation in preschoolers. Dev. Psychol. 46, 545-551. doi: 10.1037/ a0017887

Booth, J. L., and Siegler, R. S. (2006). Developmental and individual differences in pure numerical estimation. Dev. Psychol. 42, 189-201. doi: 10.1037/00121649.41.6.189

Cantlon, J. F., Platt, M. L., and Brannon, E. M. (2009). Beyond the number domain. Trends Cogn. Sci. 13, 83. doi: 10.1016/j.tics.2008.11.007

Cantrell, L., and Smith, L. B. (2013). Open questions and a proposal: a critical review of the evidence on infant numerical abilities. Cognition 128, 331-352. doi: 10.1016/j.cognition.2013.04.008

Dehaene, S., Bossini, S., and Giraux, P. (1993). The mental representation of parity and number magnitude. J. Exp. Psychol. Gen. 122, 371-396.

de Hevia, M.-D. (2011). Sensitivity to number: reply to Gebuis and Gevers. Cognition 121, 253-255. doi: 10.1016/j.cognition.2011.04.002

de Hevia, M.-D., Girelli, L., and Cassia, V. M. (2012). Minds without language represent number through space: origins of the mental number line. Front. Psychol. 3:466. doi: 10.3389/fpsyg.2012.00466

de Hevia, M.-D., and Spelke, E. S. (2009). Spontaneous mapping of number and space in adults and young children. Cognition 110, 198-207. doi: 10.1016/j.cognition.2008.11.003

de Hevia, M.-D., and Spelke, E. S. (2010). Number-space mapping in human infants. Psychol. Sci. 21, 653-660. doi: 10.1177/0956797610366091

de Hevia, M.-D., Vanderslice, M., and Spelke, E. S. (2012). Cross-dimensinal mapping of number, length and brightness by preschool children. PLoS ONE 7:e35530. doi: 10.1371/journal.pone.0035530 
Ebersbach, M. (in press). Evidence for a spatial-numerical association in kindergartners using a number line task. J. Cogn. Dev. doi: 10.1080/15248372.2013. 805134

Ebersbach, M., Luwel, K., Frick, A., Onghena, P., and Verschaffel, L. (2008). The relationship between the shape of the mental number line and familiarity with numbers in 5-to 9-year old children: evidence for a segmented linear model. J. Exp. Child Psychol. 99, 1-17. doi: 10.1016/j.jecp.2007. 08.006

Ebersbach, M., Luwel, K., and Verschaffel, L. (2013). Further evidence for a spatialnumerical association in children before formal schooling. Exp. Psychol. doi: 10.1027/1618-3169/a000250. [Epub ahead of print].

Fischer, U., Moeller, K., Bientzle, M., Cress, U., and Nuerk, H.-C. (2011). Sensorimotor spatial training of number magnitude representation. Psychon. Bull. Rev. 18, 177-183. doi: 10.3758/s13423-010-0031-3

Gebuis, T., and Gevers, W. (2011). Numerosities and space; indeed a cognitive illusion! A reply to de Hevia and Spelke (2009). Cognition 121, 248-252. doi: 10.1016/j.cognition.2010.09.008

Gevers, W., and Lammertyn, J. (2005). The hunt for SNARC. Psychol. Sci. 47, 10-21.

Gevers, W., Santens, S., Dhooge, E., Chen, Q., Van den Bossche, L., Fias, W., et al. (2010). Verbal-spatial and visuospatial coding of number-space interactions. J. Exp. Psychol. Gen. 139, 180. doi: 10.1037/a0017688

Hoffmann, D., Hornung, C., Martin, R., and Schiltz, C. (2013). Developing number-space associations: SNARC effects using a color discrimination task in 5-year-olds. J. Exp. Child Psychol. 116, 775-791. doi: 10.1016/j.jecp.2013. 07.013

Hubbard, E. M., Piazza, M., Pinel, P., and Dehaene, S. (2005). Interactions between number and space in parietal cortex. Nat. Rev. Neurosci. 6, 435-448. doi: $10.1038 / \mathrm{nrn} 1684$

Cohen Kadosh, R., Cohen Kadosh, K., Kaas, A., Henik, A., and Goebel, R. (2007). Notation-dependent and-independent representations of numbers in the parietal lobes. Neuron 53, 307-314. doi: 10.1016/j.neuron.2006.12.025

Le Corre, M., and Carey, S. (2007). One, two, three, four, nothing more: an investigation of the conceptual sources of the verbal counting principles. Cognition 105, 395-438. doi: 10.1016/j.cognition.2006.10.005

Libertus, M. E., Starr, A., and Brannon, E. M. (2014). Number trumps area for 7-month-old infants. Dev. Psychol. 50, 108-112. doi: 10.1037/a0032986

Lourenco, S. F., and Longo, M. R. (2010). General magnitude representation in human infants. Psychol. Sci. 21, 873-881. doi: 10.1177/0956797610370158

Moeller, K., Pixner, S., Kaufmann, L., and Nuerk, H.-C. (2009). Children's early mental number line: Logarithmic or decomposed linear? J. Exp. Child Psychol. 103, 503. doi: 10.1016/j.jecp.2009.02.006

Muldoon, K., Simms, V., Towse, J., Menzies, V., and Yue, G. (2011). Cross-cultural comparisons of 5-year-olds' estimating and mathematical ability. J. Cross Cult. Psychol. 42, 669-681. doi: 10.1177/0022022111406035

Nuerk, H. C., Iversen, W., and Willmes, K. (2004). Notational modulation of the SNARC and the MARC (linguistic markedness of response codes) effect. Q. J. Exp. Psychol. Sec. A 57, 835-863. doi: 10.1080/02724980343000512

Opfer, J. E., and Furlong, E. E. (2011). How numbers bias preschoolers' spatial search. J. Cross Cult. Psychol. 42, 682-695. doi: 10.1177/00220221114 06098

Opfer, J. E., and Thompson, C. A. (2006). "Even early representations of numerical magnitude are spatially organized: Evidence for a directional magnitude bias in pre-reading preschoolers," in Proceedings of the 28th Annual Conference of the Cognitive Science Society, eds R. Sun and N. Miyake (Mahwah, NJ: Erlbaum), 639-644.

Opfer, J. E., Thompson, C. A., and Furlong, E. E. (2010). Early development of spatial-numeric associations: evidence from spatial and quantitative performance of preschoolers. Dev. Sci. 13, 761-771. doi: 10.1111/j.14677687.2009.00934.x

Patro, K., and Haman, M. (2012). The spatial-numerical congruity effect in preschoolers. J. Exp. Child Psychol. 111, 534-542. doi: 10.1016/j.jecp.2011. 09.006
Proctor, R. W., and Cho, Y. S. (2006). Polarity correspondence: a general principle for performance of speeded binary classification tasks. Psychol. Bull. 132, 416. doi: 10.1037/0033-2909.132.3.416

Shaki, S., and Fischer, M. H. (2008). Reading space into numbers - a crosslinguistic comparison of the SNARC effect. Cognition 108, 590-599. doi: 10.1016/j.cognition.2008.04.001

Shaki, S., Fischer, M. H., and Göbel, S. M. (2012). Direction counts: a comparative study of spatially directional counting biases in cultures with different reading directions. J. Exp. Child Psychol. 112, 275-281. doi: 10.1016/j.jecp.2011. 12.005

Siegler, R. S., and Booth, J. L. (2004). Development of numerical estimation in young children. Child Dev. 75, 428-444. doi: 10.1111/j.1467-8624.2004. 00684.x

Siegler, R. S., and Opfer, J. E. (2003). The development of numerical estimation evidence for multiple representations of numerical quantity. Psychol. Sci. 14, 237-250. doi: 10.1111/1467-9280.02438

Slusser, E., Santiago, R., and Barth, H. (2013). Developmental change in numerical estimation. J Exp. Psychol. Gen. 142, 193-208. doi: 10.1037/a0028560

Sury, D., and Rubinsten, O. (2012). "Ordinal processing of numerical and nonnumerical information," in Reading, Writing, Mathematics and the Developing Brain: Listening to Many Voices, eds Z. Breznitz, O. Rubinsten, V. J. Molfese, and D. L. Molfese (Dordrecht: Springer), 209-232. doi: 10.1007/978-94-007-4086$0 \_13$

Szúcs, D., Nobes, A., Devine, A., Gabriel, F. C., and Gebuis, T. (2013). Visual stimulus parameters seriously compromise the measurement of approximate number system acuity and comparative effects between adults and children. Front. Psychol. 4:444. doi: 10.3389/fpsyg.2013.00444

Turconi, E., Jemel, B., Rossion, B., and Seron, X. (2004). Electrophysiological evidence for differential processing of numerical quantity and order in humans. Cogn. Brain Res. 21, 22-38. doi: 10.1016/j.cogbrainres.2004.05.003

Tversky, B., Kugelmass, S., and Winter, A. (1991). Cross-cultural and developmental trends in graphic productions. Cogn. Psychol. 23, 515-557. doi: 10.1016/0010-0285(91)90005-9

Walsh, V. A. (2003). A theory of magnitude: common cortical metrics of time, space and quantity. Trends Cogn. Sci. 7, 483-488. doi: 10.1016/j.tics.2003. 09.002

Wood, G., Willmes, K., Nuerk, H. C., and Fischer, M. H. (2008). On the cognitive link between space and number: a meta-analysis of the SNARC effect. Psychol. Sci. Q. 50, 489-525.

Wynn, K. (1990). Children's understanding of counting. Cognition 36, 155-193. doi: 10.1016/0010-0277(90)90003-3

Zebian, S. (2005). Linkages between number concepts, spatial thinking, and directionality of writing: The SNARC effect and the reverse SNARC effect in English and Arabic monoliterates, biliterates, and illiterate Arabic speakers. J. Cogn. Cult. 5, 165-190. doi: 10.1163/1568537054068660

Conflict of Interest Statement: The authors declare that the research was conducted in the absence of any commercial or financial relationships that could be construed as a potential conflict of interest.

Received: 14 May 2013; accepted: 21 April 2014; published online: 14 May 2014. Citation: Patro K, Nuerk H-C, Cress U and Haman M (2014) How numberspace relationships are assessed before formal schooling: A taxonomy proposal. Front. Psychol. 5:419. doi: 10.3389/fpsyg.2014.00419

This article was submitted to Developmental Psychology, a section of the journal Frontiers in Psychology.

Copyright (c) 2014 Patro, Nuerk, Cress and Haman. This is an open-access article distributed under the terms of the Creative Commons Attribution License (CC BY). The use, distribution or reproduction in other forums is permitted, provided the original author(s) or licensor are credited and that the original publication in this journal is cited, in accordance with accepted academic practice. No use, distribution or reproduction is permitted which does not comply with these terms. 